\title{
TUBERCULOSIS OF THE HEART
}

\author{
WITH TIIE REPORT OF TWO CASES * \\ IEDWARD WEISS, M.D. \\ PHILADELPHIA
}

In a study of 7,219 necropsies Norris ${ }^{1}$ found 1,780 tuberculous cases and among these eighty-two cases of tuberculous pericarditis. In five cases the heart muscle was involved. This indicates the relative infrequency of the conditions being reported. A point of added interest is the unusual degree of involvement in the first case.

\section{REPORT OF CASES}

CASE 1.-History.-The patient, a colored male, aged 25, was admitted to the Jefferson Hospital, on the service of Dr. H. A. Hare, Dec. 27, 1920, complaining of pain in the upper half of the abdomen and chest. His family history was negative for tuberculosis. He had gonorrhea in 1909 and syphilis in 1913, for which he received no treatment. In October, 1918, he had an attack of influenza and since then a persistent cough at night. In July, 1920. he developed abdominal pain which grew progressively worse and about December, 1920, his abdomen began to enlarge. His cough became more severe; he was dyspneic and complained of pain in his chest. He stated that he had lost 40 pounds in the past year.

Examination.-On examination there were evidences of a pleural effusion at the right base and immense enlargement of the heart - both confirmed by roentgen ray. His abdomen was slightly distended and tender and the liver could be palpated at the level of the umbilicus in the right midclavicular line. There was some fluid in the left tunica vaginalis. Blood count showed a leukopenia and moderate secondary anemia; his blood Wassermann was positive; his sputum was negative for tubercle bacilli. He died Feb. 21, 1921, and necropsy was performed the same day.

Necropsy Report.-The body was that of an adult colored male, weighing about 170 pounds. The heart and pericardium were immensely enlarged, weighing 1,580 $\mathrm{gm}$., the pericardial cavity being obliterated by large, dense, yellowish nodules which invaded the heart muscle, auricles and ventricles to a similar degree. The nodules were continuous with the mediastinal and peribronchial lymph nodes which were also large, yellowish and firm. No tuberculosis of the lungs could be established but the pleurae averaged about $0.4 \mathrm{~cm}$. in thickness and this thickening was especially marked at the right base which contained an encapsulated effusion of about 700 c.c. of brownish-red serum. The spleen, right suprarenal, kidneys and liver showed a number of firm, yellowish nodules scattered throughout. At the juncture of the ileum and cecum was a large ulcer, $2.5 \mathrm{~cm}$. in diameter, with base of reddish granulations and firm, undermined edges. This, with the large, firm and yellowish mesenteric and retroperitoneal nodes aided in the gross diagnosis of tuberculosis.

The microscopic study revealed a fibrocaseous tuberculosis of the following organs and tissues: pericardium and myocardium, pleurae, spleen, right adrenal, kidneys, liver, mediastinal, mesenteric and retroperitoneal lymph nodes.

* Read before the Pathological Section of the National Tuberculosis Association, New York City, June 16, 1921.

* From the Department of Pathology, Jefferson Medical College.

1. Norris, G. W.: Tulerculous Pericarditis Based on a Study of 7,219 Autopsies in Philadelphia Hospitals, Univ. Penn. M. Bull. 17:155, 1904. 
No typical tubercles were scen and very few giant cells. Smears from the fresh material and stained sections of pericardium. pleura and retroperitoneal nodes were studied for tubercle bacilli but failed to reveal the organism. Guinea-pig inoculation, however, produced a diffuse tulserculous lymphadenitis and tuberculosis of the splecn. Smears from the caseous nodes showed many tuhercle bacilli but attempts to culture the organism failed.

CASE 2.-S. W., a colored male, aged 25, dishwasher by occupation, was admitted to the Department for Diseases of the Chest of the Jefferson Hospital, March 15, 1921." He complained of a dull pain in the lack and sternal region and of a moderate cough productive of a large amount of mucoid sputum which was occasionally blond-streaked.

Family History.-Negative for tuberculosis.

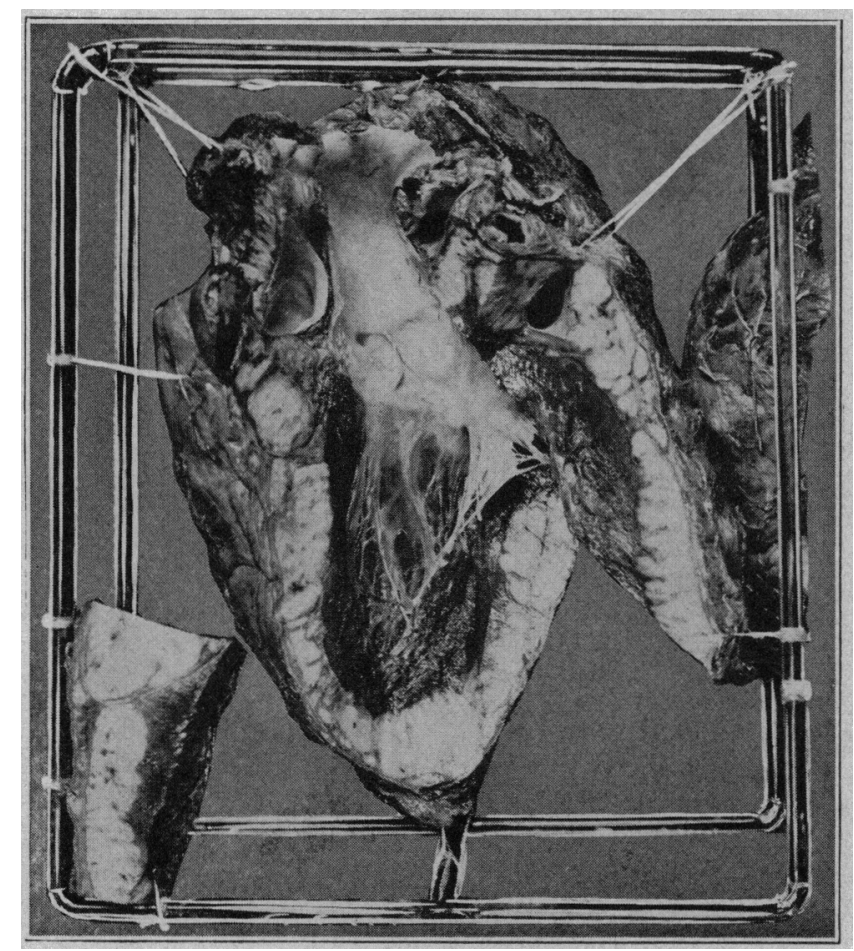

Fig. 1.-Case 1. Tuberculous pericarditis and myocarditis. Note the invasion of heart muscle by the immense, tuberculous nodules of the pericardium. shown lest by insert in left hand corner.

Personal History.-He had a chancre in 1913 and gonorrhea in 1914 . He stated that he had been in poor health ever since his discharge from the army in 1918. At that time he noticed a cough. Shortly after the onset he became short of breath on slight exertion and felt weak. From time to time he had a vague pain in the front of the chest. Increasing disability caused him to give up his work in the latter part of January, 1921. He entered a Philadelphia hospital and was later transferred to the Jefferson Chest Hospital.

2. I am indebted to Dr. E. H. Funk for the clinical notes of this case. 
Physical Examination.-This showed an emaciated, colored, adult male. His pupils reacted normally. Throat was red, tonsils swollen. The cervical lymph nodes were distinctly palpable with a small mass of enlarged glands above the clavicle on the left side. Chest was long, narrow and flat. Expansion was generally limited. Vocal fremitus was increased throughout the right side of the chest and the percussion note was generally impaired. Breath sounds were distinctly audible with a blowing characteristic over the entire chest except the right base posteriorly where the intensity was diminished. Many squeaking sounds were heard with numerous crackling râles throughout, especially

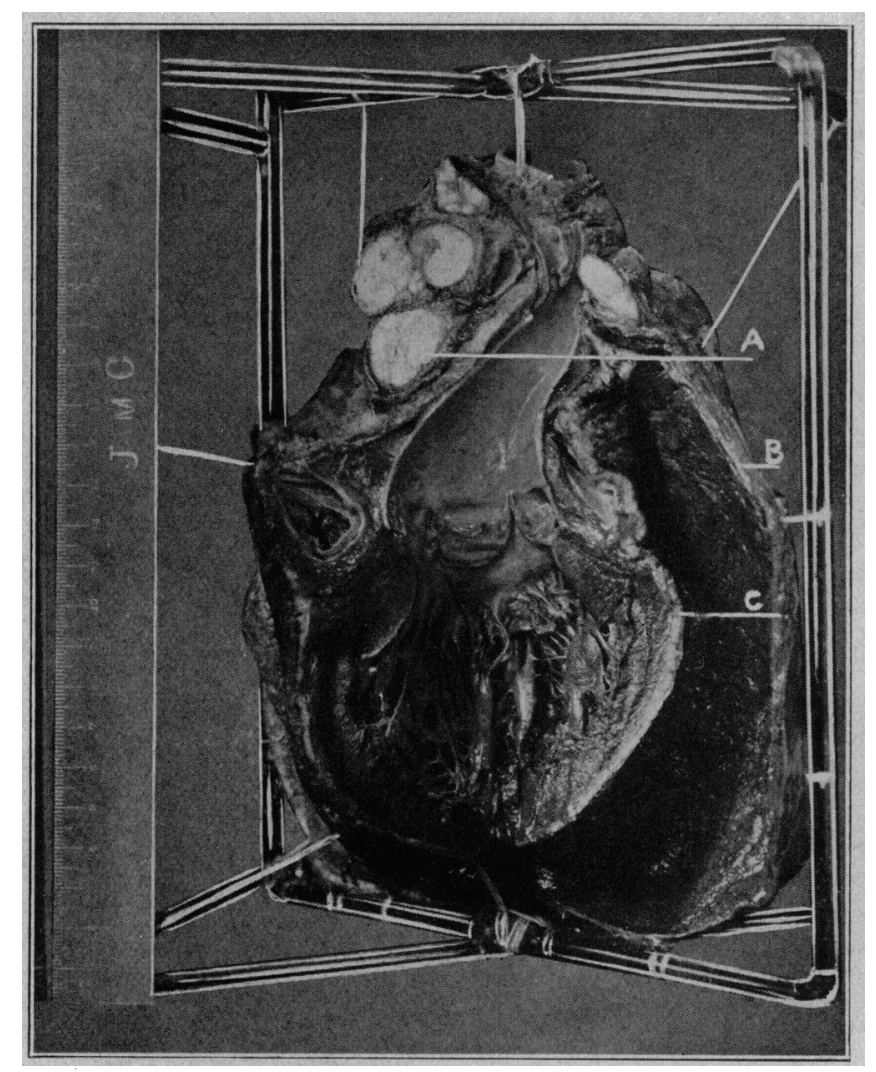

Fig. 2.--Case 2. Tuberculous pericarditis, showing (a) enlarged, tuberculous nodes at the base of the heart; (b) thickened, parietal pericardium, and (c) tuberculous nodules of visceral pericardium.

on the right side. Whispering pectoriloquy was noted in the third interspace on the right side, anteriorly.

Heart: There was marked precordial pulsation with an increase to the left in the lateral diameter of cardiac dulness. Heart action was rapid but regular; heart sounds were distinctly heard; no murmurs were present. Sputum examinations were repeatedly negative for tubercie hacilli. Urine contained a trace of albumin and an occasional hyaline cast. Temperature, $102 \mathrm{~F}$.; pulse, 130; respirations, 40. Patient died, April 25, 1921, and a necropsy was made the following day. 
Necropsy Report.-The important findings were as follows: The anterior cervical lymph nodes were distinctly palpable and those on the left. side just above the clavicle were especially enlarged. There werc several enlarged, firm, yellowish nodes adherent to the posterior surface of the sternum. All of the mediastinal nodes were similarly enlarged, clustcred about and pressing on the trachea and larger bronchi. These nodes varied in size from less than $1 \mathrm{~cm}$. to 3 or $4 \mathrm{~cm}$. in diameter. Some had caseous centers.

The pericardial cavity was distended with about 700 c.c. of blood-tinged serous fluid. There was likewise a dense, yellowish, fibrinous exudate present.

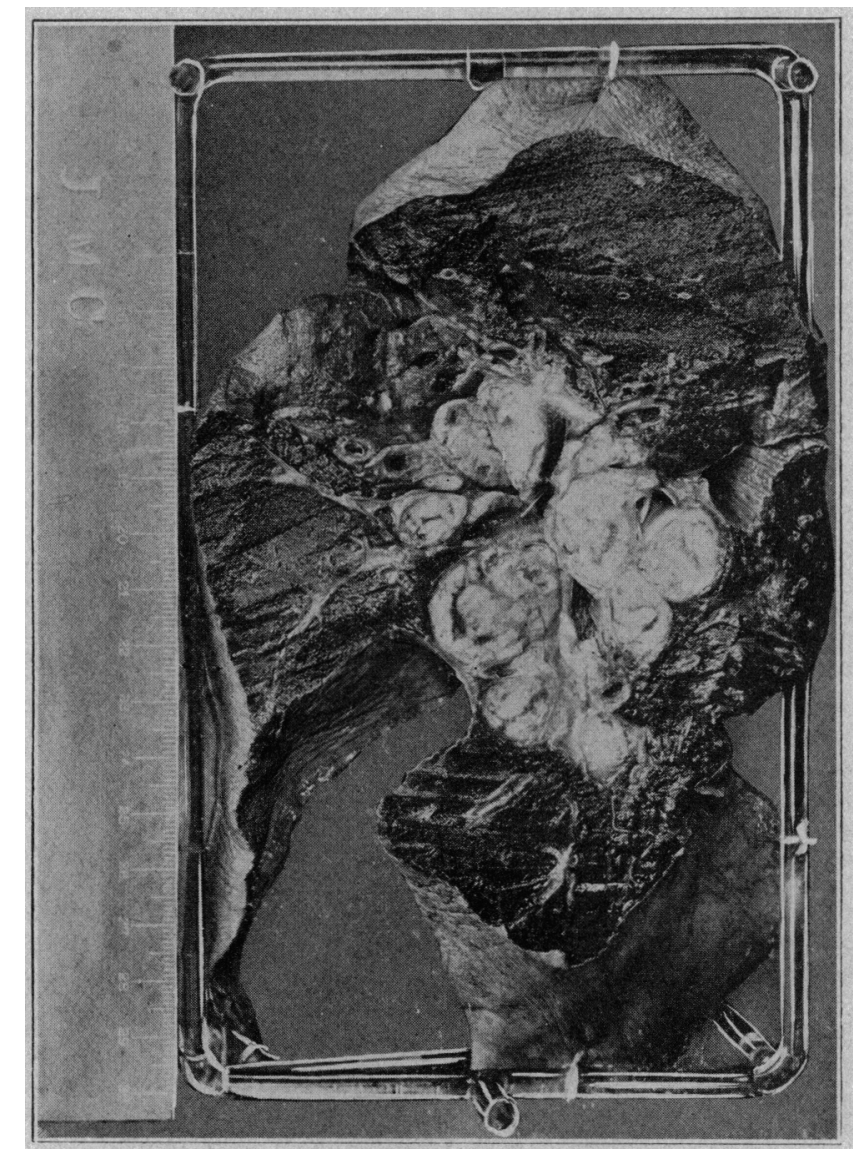

Fig. 3.-Case 2. Tuberculosis of peribronchial lymph nodes with practically no lung involvement.

The visceral pericardium of the left ventricle was distinctly thickened, measuring about $0.3 \mathrm{~cm}$. , and consisted of a zone of dense, yellowish tissue made up of conglomerate nodules which grew larger near the base of the ventricle at the reflection of the pericardium. These nodules, unlike those of the first case. slowed no tendency to invade the heart muscle. The lymph nodes at the base of the heart were of the same type as the mediastinal nodes and were continuous with them, pressing on the great vessels. These nodes were likewise firmly adherent to the pericardium. 
Left Lung: The left pleural cavity contained about 500 c.c. blood-tinged serous fluid and the lung was compressed. On section the pulmonary tissue was dark red in color and only partially crepitant. About $6 \mathrm{~cm}$. from the apex, on the anterolateral surface of the lung, was a conglomerate caseous tubercle, a little less than a centimeter in diameter. There was no further evidence of tuberculosis in the lung structure; the nodes at the hilus, however, were greatly enlarged, similar to and continuous with the mediastinal nodes.

Right Lung: It was more voluminous than the left but on section presented nearly the same appearance. Near the hilus, a little area of pulmonary tissue, about $2 \mathrm{~cm}$. in diameter, adjacent to an enlarged and caseous lymph node, showed tuherculous infiltration.

None of the other organs showed any evidence of tuberculosis but the mesenteric and retroperitoneal lymph nodes were moderately enlarged, firm and yellowish.

A few tubercle bacilli were found in smears from the caseous mediastinal nodes.

Microscopic study revealed a typical caseous tuberculosis of the structures mentioned. The pericardium showed the lesion especially well; unlike the first case, there were many typical tubercles present with caseous centers and numerous giant cells. The tuberculous process, as mentioned in the gross description, exhibited no tendency to invade the myocardium.

Guinea-pigs were inoculated with emulsified caseous nodules from the base of the heart. The animals, killed six weeks later, showed tuberculosis of the abdominal lymph nodes and spleen. A number of tubercle bacilli were recovered in smears but cultures both on Petroff's and Dorset's mediums failed to develop the organism.

COM MENT

In 1902 Anders $^{3}$ stummarized seventy-one cases of tuberculosis of the myocardium and added a case of his own. He classified the condition as occurring in three forms: (1) large tubercles; (2) miliary tubercles (less common) and (3) diffuse form or tuberculous infiltratiton (rare). He stated that cardiac tuberculosis was most frequent in early life, 40 per cent. of his cases occurring under 15 years. In a large proportion of cases, the lungs and bronchial glands were tuberculous. In twenty-nine out of thirty-one cases in which the glandular condition was noted, tuberculosis was present and this he believed to be the seat of the disease. This extended from the bronchial to the mediastinal nodes and thence directly to the heart or quite commonly by way of the pericardium.

Cases very similar to the present ones have been reported by Ellis, Raviart and Caudron, ${ }^{5}$ Benda and Geissler, ${ }^{6}$ Toldt, ${ }^{7}$ Passamonti, ${ }^{8}$

3. Anders, J. M.: Tuberculosis of the Myocardium, J. A. M. A. 39:1081 (Nov. 1) 1902.

4. Ellis, A. G.: Heart Showing Chronic Tuberculosis of the Pericardium, with Involvement of the Myocardium, Proc. Path. Soc., Phila., June. 1903.

5. Raviart and Caudron: A Case of Tuberculosis of the Myocardium, Echo méd. du Nord 8:529, 1904.

6. Benda, C., and Geissler: New Cases of Tuberculosis of the Heart and Blood Vessels. Deutsch. med. Wchnschr. 31:1169 (July 20) 1905.

7. Toldt, G.: A Case of Tuberculosis of the Myocardium, Rev de méd. 26: $101,1906$.

8. Passamonti. M.: A Case of Tuberculosis of the Myocardium, Policlinico, Rome 14:88, 1907. 
Beifeld, ${ }^{9}$ Fraga, ${ }^{10}$ and more recently by Adamson, ${ }^{11}$ Doermer, ${ }^{12}$ and Binder. ${ }^{13}$

Ellis' case presented, in addition to tuberculous pericarditis and myocarditis, tuberculosis of the mediastinal nodes and lungs and a miliary tuberculosis of liver, spleen, pancreas and kidneys. Only after a study of many blocks from the heart were a few tubercle bacilli seen in one section. Beifeld's case showed a tuberculous scar at the apex of the left lung and a caseous tuberculosis of the mediastinal, tracheal, bronchial and left lower cervical nodes. There was a tuberculous pericarditis with total obliteration of the pericardial sac. The liver and spleen contained conglomerate tubercles. Microscopic examination demonstrated typical tuberculous tissue but up to the time of his report search for tubercle bacilli in the tissues was unsuccessful. He concluded that infection traveled from the left apex to the mediastinal nodes and then by contiguity or lymphatic extension or both, to the pericardium and by contact infection to the heart muscle. In Adamson's case no evidence of tuberculosis was found in the lungs or bronchial lymph nodes but he felt that the process probably started in the nodes at the base of the heart.

It is generally conceded that the mediastinal nodes act as the focus of infection for the pericardium and myocardium in this condition. Toldt however, reports a case of tuberculosis of the heart (limited to the right auricle) with no sign of pericardial involvement. He found but little evidence of tuberculosis disease in the tracheobronchial nodes but did note a healed lesion at the apices of both lungs. In commenting on the condition he mentions that Fuchs in a report of fifty-three cases of tuberculosis of the myocardium, found twelve cases of isolated auricular tuberculosis. Of these the right auricle alone was affected in nine; both auricles in two; and the left auricle only in one. Of the twelve cases eight showed tuberculous pericarditis, in two the pericardium was unaffected and in two the condition was not stated.

Passamonti also reports a case of tuberculosis of the myocardium with no other tuberculous lesion except a nodule in the lower lobe of the left lung. He did not feel that the usual propagation of infection (glandular) had occurred in his case. In a discussion of the condition

9. Beifeld. A. F.: Tulerculosis of the Myocardium, with the Report of a Case, Tr. Chicago Path. Soc. 8:104, 1909-1912.

10. Fraga, C.: Concerning a Case of Cardio-Tuberculous Cirrhosis, Brazilmed. 31:398, 1917.

11. Adamson, W. W.: A Case of Tuberculosis of the Myocardium. J. Path. \& Bacteriol. 23:399 (Dec.) 1920.

12. Doermer, W.: A Case of Conglomerate Tuberculosis of the heart, Diss., Jena, 1918.

13. Binder, A.: Tumor-Like Tuberculosis of the Heart, Zentralbl, f, inn. Med. 41:462, 1920. 
he mentions that the finding is practically always a postmortem one, and from a study of the literature brings out the fact that there are no characteristic signs of tuberculosis of the heart. He states that attacks of dyspnea, cyanosis, arrhythmia and enlargement of the cardiac area have been observed but adds that hypertrophy and dilatation are not always noted. Raviart and Caudron record a case in a white male, aged 31, with vague symptoms of oppression and pain in the epigastrium, and no definite physical signs. Necropsy revealed a tracheobronchial glandular tuberculosis and tuberculosis of the heart (confined to the right auricle). Toldt's case, mentioned above, occurred in a woman of 58 who had been ill for eighteen months, but, with "no symptoms of localization." Examination showed deviation of the apex of the heart, arrhythmia and muffled apical meurmurs-no other physical signs.

Anders made note of the following symptoms: palpitation, feeble heart sounds, pericardial distress, diffuse pulsation, tumultuous and rapid heart action, fetal and gallop rhythym, rarely murmurs, sudden and recurring syncope, dyspnea, cyanosis, unconsciousness, general edema and sudden death. But he states, "in all this list there is nothing specific, no single symptom or combination of symptoms that is not seen in functional and organic disease of the heart other than tuberculosis. Often the patient dies without a symptom that would attract special attention to the heart." Eisenmeyer is quoted by Anders as stating that a diagnosis may occasionally be made by the presence in a victim of general tuberculosis of sudden, severe collapse, quickly passing; and the detection of weak endocardial murmurs varying in phase and intensity.

One point not mentioned in the cases cited, is the apparently, relatively frequent occurrence of this unusual form of tuberculosis in the colored race. Four cases, Ellis', Beifeld's and the two making up the present report, occurred in young, adult, colored males. For this reason it seems reasonable to suggest that when evidence of cervical and mediastinal glandular disease exists in a young colored man with indefinite symptons referable to the circulatory system and possibly signs of cardiac enlargement-tuberculosis of the heart should certainly be considered. In this connection Biefeld's case merits particular mention. A colored male, aged 21, had dyspnea and cyanosis; cough; rapid, regular pulse of good quality; and an evening rise of temperature. Examination showed a heart enlarged to right and left. No murmurs were present but a systolic retraction was noted, in place of the apex beat. A clinical diagnosis was made of adherent pericardium, tuberculous in nature; the necropsy, as above described, confirmed this diagnosis. 
SUMMARY

The brief clinical reports and necropsy findings of two colored, young, adult males are recorded. Symptoms and physical signs were vague; necropsy demonstrated an immense, fibrocaseous tuberculous involvement of the lymph nodes of the thorax, with extension to the pericardium and in the one case to the heart muscle, causing tremendous enlargement of the organ. Reference to the literature indicates that the heart involvement is almost always secondary to the disease of the mediastinal lymph nodes. The practical limitation of the process to the lymph nodes, the curious reaction of the tissues and the difficulties in the diagnosis of such conditions are some of the problems offered to pathologist and clinician by these case reports.

I am indebted to Dr. H. A. Hare and Dr. Thomas McCrae for permission to report these cases. 